DOI: $10.17516 / 1997-1397-2021-14-5-573-583$

УДК 519.218.2+517.518.26

\title{
On Estimation of the Convergence Rate to Invariant Measures in Markov Branching Processes with Possibly Infinite Variance and Immigration
}

\author{
Azam A. Imomov* \\ Karshi State University \\ Karshi city, Uzbekistan
}

Received 31.03.2021, received in revised form 29.05.2021, accepted 20.06.2021

\begin{abstract}
The continuous-time Markov Branching Process with Immigration is discussed in the paper. A critical case wherein the second moment of offspring law and the first moment of immigration law are possibly infinite is considered. Assuming that the non-linear parts of the appropriate generating functions are regularly varying in the sense of Karamata, theorems on convergence of transition functions of the process to invariant measures are proved. The rate of convergence is determined provided that slowly varying factors are with remainder.
\end{abstract}

Keywords: Markov branching process, generating functions, immigration, transition functions, slowly varying function, invariant measures, convergence rate.

Citation: A.A.Imomov, On Estimation of the Convergence Rate to Invariant Measures in Markov Branching Processes with Possibly Infinite Variance and Allowing Immigration, J. Sib. Fed. Univ. Math. Phys., 2021, 14(5),573-583. DOI: 10.17516/1997-1397-2021-14-5-573-583.

\section{Introduction and preliminaries}

The discussion of the population growth model called the continuous-time Markov Branching Process with Immigration (MBPI) which was considered in [5] is continued in this paper. Recall that this process has simple physical interpretation: the population size changes not only as a result of reproduction and disappearance of existing individuals but also as a result of the random influx of "extraneous" individuals of the same type from the outside. Namely, the process develops according to the following scheme. Each individual existing at time $t \in \mathcal{T}:=[0,+\infty)$ independently of his history and of each other for a small time interval $(t, t+\varepsilon)$ is transformed into $j \in \mathbb{N}_{0} \backslash\{1\}$ individuals with probability $a_{j} \varepsilon+o(\varepsilon)$, and with probability $1+a_{1} \varepsilon+o(\varepsilon)$ stays to live or makes evenly one descendant (as $\varepsilon \downarrow 0$ ). Here $\mathbb{N}_{0}=\{0\} \cup \mathbb{N}$ and $\mathbb{N}$ is the set of natural numbers, and $\left\{a_{j}\right\}$ are intensities of individual transformation, $a_{j} \geqslant 0$ for $j \in \mathbb{N}_{0} \backslash\{1\}$ and $0<$ $a_{0}<-a_{1}=\sum_{j \in \mathbb{N}_{0} \backslash\{1\}} a_{j}<\infty$. Independently of these for each time interval $j \in \mathbb{N}$ new individuals enter the population with probability $b_{j} \varepsilon+o(\varepsilon)$, and immigration does not occur with probability $1+b_{0} \varepsilon+o(\varepsilon)$. Immigration intensities $b_{j} \geqslant 0$ for $j \in \mathbb{N}$ and $0<-b_{0}=\sum_{j \in \mathbb{N}} b_{j}<\infty$. Newly arrived individuals undergo transformation in accordance with the reproduction law generated by intensities $\left\{a_{j}\right\}$; see [11, p. 217]. Thus, the process under consideration is completely determined by infinitesimal generating functions $(\mathrm{GFs})$

$$
f(s):=\sum_{j \in \mathbb{N}_{0}} a_{j} s^{j} \quad \text { and } \quad g(s):=\sum_{j \in \mathbb{N}_{0}} b_{j} s^{j} \quad \text { for } s \in[0,1) .
$$

*imomov_azam@mail.ru https://orcid.org/ 0000-0003-1082-0144 (c) Siberian $\overline{\text { Federal University. All rights reserved }}$ 
Let us denote the population size at the time $t \in \mathcal{T}$ in MBPI by $X(t)$. This is homogeneous continuous-time Markov chain with state space $\mathcal{S} \subset \mathbb{N}_{0}$ and transition functions

$$
p_{i j}(t):=\mathbb{P}_{i}\{X(t)=j\}=\mathbb{P}\{X(t+\tau)=j \mid X(\tau)=i\}
$$

for all $i, j \in \mathcal{S}$ and $\tau, t \in \mathcal{T}$.

Only critical case is considered in the paper, i.e., $f^{\prime}(1-)=\sum_{j \in \mathbb{N}} j a_{j}=0$, and limit behaviours of transition functions $p_{i j}(t)$ as $t \rightarrow \infty$ is observed. Pakes [9] was one of the first who studied invariant measures for MBPI with finite variance and found an integral form of GF of invariant measures. He has proved that limits $\pi_{j}:=\lim _{t \rightarrow \infty} t^{\lambda} p_{i j}(t)$ exist independently on $j$, iff $\sum_{j \in \mathbb{N}} a_{j} j^{2} \ln j<$ $\infty$ and $\sum_{j \in \mathbb{N}} b_{j} j \ln j<\infty$, where $\lambda=2 g^{\prime}(1-) / f^{\prime \prime}(1-)$, besides the set $\left\{\pi_{j}, j \in \mathcal{S}\right\}$ presents an invariant measure for MBPI. The invariant measure of MBPI can also be constructed by the strong ratio limit property of transition functions but slightly different [7]. Namely, the set of positive numbers $\left\{v_{j}:=\lim _{t \rightarrow \infty} p_{0 j}(t) / p_{00}(t)\right\}$ is an invariant measure. Moreover one can see a close relation between the sets $\left\{\pi_{j}, j \in \mathcal{S}\right\}$ and $\left\{v_{j}, j \in \mathcal{S}\right\}$, and their GFs $\pi(s)=\sum_{j \in \mathcal{S}} \pi_{j} s^{j}$ and $\mathcal{U}(s)=\sum_{j \in \mathcal{S}} v_{j} s^{j}$. In fact, they are really only different versions of the same limit law. So, it is easy to see that $\mathcal{U}(s)=\pi(s) / \pi(0)$, and this is consistent with uniqueness, up to a multiplicative constant, of the invariant measure of MBPI.

An estimation of the rate of convergence to invariant measures is of exceptional interest. The rate of convergence of $t^{\lambda} p_{i j}(t)$ to $\pi_{j}$ for all $i, j \in \mathcal{S}$ was studied under the condition $\max \left\{f^{\prime \prime \prime}(1-), g^{\prime \prime}(1-)\right\}<\infty[5]$. It was found that the convergence rate is $\mathcal{O}(\ln t / t)$ as $t \rightarrow \infty$.

Throughout the paper, the following Basic assumptions for $f(s)$ and $g(s)$ are used

$$
f(s)=(1-s)^{1+\nu} \mathcal{L}\left(\frac{1}{1-s}\right),
$$

and

$$
g(s)=-(1-s)^{\delta} \ell\left(\frac{1}{1-s}\right)
$$

for all $s \in[0,1)$, where $0<\nu, \delta<1$ and $\mathcal{L}(\cdot), \ell(\cdot)$ are slowly varying at infinity $\left(\mathbf{S V}_{\infty}\right)$ in the sense of Karamata (see, for instance, [2] and [10]). Basic assumptions imply that the offspring distribution belongs to the domain of attraction of the $(1+\nu)$-stable law, and the immigration distribution belongs to the domain of attraction of the $\delta$-stable law. In the critical case assumption $\left[f_{\nu}\right]$ implies that $2 b:=f^{\prime \prime}(1-)=\infty$. If $b<\infty$ then representation $\left[f_{\nu}\right]$ holds with $\nu=1$ and $\mathcal{L}(t) \rightarrow b$ as $t \rightarrow \infty$. Similarly, GF $g(s)$ of the form $\left[g_{\delta}\right]$ generates the immigration law with the $\delta$-order moment. However, if $g^{\prime}(1-)<\infty$ then assumption $\left[g_{\delta}\right]$ is fulfilled with $\delta=1$ and $\ell(t) \rightarrow g^{\prime}(1-)$ as $t \rightarrow \infty$.

An additional requirement for $\mathcal{L}(x)$ and $\ell(x)$ is introduced:

$$
\frac{\mathcal{L}(\lambda x)}{\mathcal{L}(x)}=1+\mathcal{O}(\alpha(x)) \quad \text { as } x \rightarrow \infty
$$

for each $\lambda>0$, where $\alpha(x)$ is known positive decreasing function so that $\alpha(x) \rightarrow 0$ as $x \rightarrow \infty$. In this case $\mathcal{L}(x)$ is called $\mathrm{SV}_{\infty}$ with remainder $\mathcal{O}(\alpha(x)$ ) (see [2, p. 185, condition SR1]). When employing condition $\left[\mathcal{L}_{\nu}\right]$ it is assumed that

$$
\alpha(x)=\mathcal{O}\left(\frac{\mathcal{L}(x)}{x^{\nu}}\right) \quad \text { as } x \rightarrow \infty .
$$


Similarly, the condition

$$
\frac{\ell(\lambda x)}{\ell(x)}=1+\mathcal{O}(\beta(x)) \quad \text { as } x \rightarrow \infty
$$

is also allowed for each $\lambda>0$, where

$$
\beta(x)=\mathcal{O}\left(\frac{\ell(x)}{x^{\delta}}\right) \quad \text { as } x \rightarrow \infty .
$$

It was shown that the asymptotes of the transition functions depend on the sign of the parameter $\gamma:=\delta-\nu[3]$. In addition, the limit functions $U(s):=\lim _{t \rightarrow \infty} \mathcal{P}(t ; s)$ for $\gamma>0$ and $\pi(s):=\lim _{t \rightarrow \infty} e^{T(t)} \mathcal{P}(t ; s)$ for $\gamma<0$ and for some $T(t)$ were found. hold.

In this paper the rate of convergence is determined provided that conditions $\left[\mathcal{L}_{\nu}\right]$ and $\left[\ell_{\delta}\right]$

The rest of this paper is organized as follows. Section 2. contains main results. Auxiliary statements that are used in the proof of theorems are considered in Section 3.. Proof of main results is presented in Section 4. .

\section{Main results}

Let us consider $\operatorname{GF} \mathcal{P}_{i}(t ; s):=\sum_{j \in \mathcal{S}} p_{i j}(t) s^{j}$. It is not difficult to see that (see [9])

$$
\mathcal{P}_{i}(t ; s)=(F(t ; s))^{i} \exp \left\{\int_{0}^{t} g(F(u ; s)) d u\right\},
$$

where $F(t ; s)$ is GF of Markov Branching Process initiated by single individual without immigration. Since $F(t ; s) \rightarrow 1$ as $t \rightarrow \infty$ uniformly in $s \in[0, d], d<1$ (see Lemma 1 below), it is sufficient to consider $\mathcal{P}(t ; s):=\mathcal{P}_{0}(t ; s)$. Then taking into account Basic assumptions and the Kolmogorov backward equation $\partial F / \partial t=f(F)$, it follows from (1) that

$$
\mathcal{P}(t ; s)=\exp \left\{\int_{s}^{F(t ; s)} \frac{g(u)}{f(u)} d u\right\}
$$

Taking into account Basic assumptions, the integrand is

$$
\frac{g(u)}{f(u)}=-(1-u)^{\gamma-1} L\left(\frac{1}{1-u}\right)
$$

where $\gamma:=\delta-\nu$ and

$$
L(t):=\frac{\ell(t)}{\mathcal{L}(t)}
$$

State space $\mathcal{S}$ can be classified in accordance with the sign of $\gamma$. By virtue of (3), integral

$\int_{s}^{1}[g(u) / f(u)] d u$ converges if $\gamma>0$, and diverges if $\gamma<0$. It was shown that $\mathcal{S}$ is positiverecurrent if $\gamma>0$, and it is transient if $\gamma<0$. The special case $\gamma=0$ implies that $g(s)=f^{\prime}(s)$ and $L(t) \rightarrow 1+\nu$ as $t \rightarrow \infty$. It is another population process called Markov Q-process (see [4], [6], [1, pp. 56-58] and [8] for the discrete-time case).

Main results are formulated only for the case $\gamma \neq 0$ in the following two theorems. Let

$$
\tau(t):=\frac{(\nu t)^{1 / \nu}}{\mathcal{N}(t)} \quad \text { and } \quad T(t):=(\tau(t))^{|\gamma|},
$$

where $\mathcal{N}(x)$ is $\mathrm{SV}_{\infty}$ defined in Lemma 1 below. 
Theorem 2.1. Let $\gamma>0$. Then $\mathcal{P}(t ; s)$ converges to the function $U(s)=$ $=\exp \left\{\int_{s}^{1}[g(u) / f(u)] d u\right\}$ for $s \in[0,1)$, and its power series expansion $U(s)=\sum_{j \in \mathcal{S}} u_{j} s^{j}$ generates an invariant distribution $\left\{u_{j}, j \in \mathcal{S}\right\}$ for $M B P I$. The convergence is uniform over compact subsets of $[0,1)$. In addition, if assumptions $\left[\mathcal{L}_{\nu}\right]$ and $\left[\ell_{\delta}\right]$ hold then

$$
\mathcal{P}(t ; s)=U(s)(1+\Delta(t ; s) \mathcal{K}(\tau(t)))
$$

where $\mathcal{K}(x)=\mathcal{L}^{-\delta / \nu}(x) \ell(x)$, function $\mathcal{N}(x)$ is $S V_{\infty}$ defined in (13) below and

$$
\Delta(t ; s)=\frac{1}{\gamma} \frac{1}{(\lambda(t ; s))^{\gamma / \nu}}+\mathcal{O}\left(\frac{\ln [\Lambda(1-s) \lambda(t ; s)]}{(\lambda(t ; s))^{\delta / \nu}}\right) \quad \text { as } t \rightarrow \infty,
$$

where $\lambda(t ; s)=\nu t+\Lambda^{-1}(1-s)$ and $\Lambda(y)=y^{\nu} \mathcal{L}(1 / y)$. The transition functions are

$$
p_{i j}(t)=u_{j}\left(1+\mathcal{O}\left(\frac{K(t)}{t^{\gamma / \nu}}\right)\right) \quad \text { as } t \rightarrow \infty
$$

where $K(t)$ is $S V_{\infty}$.

Another asymptotic property comes out for $\mathcal{P}(t ; s)$ when $\gamma<0$. Taking into account Basic assumptions, one can easily verify that

$$
-\frac{\ln p_{00}(t)}{T(t)} \sim \frac{1}{|\gamma|} L(\tau(t)) \quad \text { as } t \rightarrow \infty
$$

This asymptotic relation shows that $(T(t))^{-1} \ln p_{00}(t)$ is asymptotically $\mathrm{SV}_{\infty}$. Then one should consider the limit of the function $e^{T(t)} \mathcal{P}(t ; s)$ as $t \rightarrow \infty$. First one needs to consider $\mathrm{SV}_{\infty}$ property of $L(t)$. In accordance with the slowly varying theory, functions $\ell(\cdot)$ and $\mathcal{L}(\cdot)$ are positive. Then by virtue of $[2$, p. 185, Theorem 3.12.2 (SR1)], one can obtain the following propositions:

$$
\begin{aligned}
& \text { - }\left[\mathcal{L}_{\nu}\right] \quad \Longleftrightarrow \quad \mathcal{L}(x)=C_{\mathcal{L}}+\mathcal{O}(\alpha(x)) \quad \text { as } t \rightarrow \infty, \\
& \text { - }\left[\ell_{\delta}\right] \quad \Longleftrightarrow \quad \ell(x)=C_{\ell}+\mathcal{O}(\beta(x)) \quad \text { as } t \rightarrow \infty,
\end{aligned}
$$

where $C_{\mathcal{L}}, C_{\ell}$ are positive constants and functions $\alpha(x), \beta(x)$ are in $\left[\mathcal{L}_{\nu}\right]$ and $\left[\ell_{\delta}\right]$. Then

$$
L(t)=\frac{\ell(t)}{\mathcal{L}(t)}=C_{L}+\mathcal{O}\left(\frac{\ell(t)}{t^{\delta}}\right) \quad \text { as } t \rightarrow \infty,
$$

since $\delta<\nu$, where $C_{L}=C_{\ell} / C_{\mathcal{L}}$. This requirement for $L(t)$ is quite possible. Especially, one can obtain an "excellent result" if $C_{L}=|\gamma|$ is chosen. The following explicit form of $\pi(s)=$ $\lim _{t \rightarrow \infty} e^{T(t)} \mathcal{P}(t ; s)$ was found in [3]:

$$
\pi(s)=\exp \left\{\frac{1}{(1-s)^{|\gamma|}}+\int_{s}^{1}\left[\frac{g(u)}{f(u)}+\frac{|\gamma|}{(1-u)^{1+|\gamma|}}\right] d u\right\} .
$$

Now the convergence rate of $e^{T(t)} \mathcal{P}(t ; s)$ to $\pi(s)$ is determined in the following theorem.

Theorem 2.2. Let $\gamma<0$ and $C_{L}=|\gamma|$ in (6). If $\mu:=2 \delta-\nu>0$ then

$$
e^{T(t)} \mathcal{P}(t ; s)=\pi(s)(1+\rho(t ; s)),
$$


where $\rho(t ; s) \rightarrow 0$ as $t \rightarrow \infty$ uniformly in $s \in[0, r], r<1$, and the limiting $G F \pi(s)$ can be expressed in the form given in $(7)$. In addition, if assumptions $\left[\mathcal{L}_{\nu}\right]$ and $\left[\ell_{\delta}\right]$ hold then

$$
\rho(t ; s)=\mathcal{O}\left(\frac{\ell(\tau(t))}{(\tau(t))^{\mu}}\right) \quad \text { as } t \rightarrow \infty
$$

uniformly in $s \in[0, r], r<1$. Denoting the power series expansion of $\pi(s)$ by $\sum_{j \in \mathcal{S}} \pi_{j} s^{j}$, transition functions have the form

$$
p_{i j}(t)=\pi_{j}\left(1+\mathcal{O}\left(\frac{\ell(\tau(t))}{(\tau(t))^{\mu}}\right)\right) \quad \text { as } t \rightarrow \infty
$$

and $\left\{\pi_{j}, j \in \mathcal{S}\right\}$ is an invariant measure for $M B P I$.

Remark 1. The form of limiting GF $\pi(s)$ given in the first part of Theorem 2.2 is compatible with the results presented in [9] and [5] where the case $\max \left\{f^{\prime \prime}(1-), g^{\prime}(1-)\right\}<\infty$ was considered. Thus, this theorem essentially strengthens last-mentioned results.

Remark 2. The conditions $C_{L}=|\gamma|$ and $\mu>0$ in Theorem 2.2 are essential because they ensure the convergence of the integral in (7). In fact, due to Basic assumptions and (6) the majorizing function for the integrand is $(1-u)^{\mu-1}$. Then function

$$
\mathcal{B}(s):=\exp \left\{\int_{s}^{1}\left[\frac{g(u)}{f(u)}+\frac{|\gamma|}{(1-u)^{1+|\gamma|}}\right] d u\right\}
$$

is bounded for $s \in[0,1]$.

The following result is a consequence of Theorem 2.2.

Corollary 1. Under the conditions of Theorem 2.2

$$
e^{T(t)} p_{00}(t)=\mathcal{B}(0)\left(1+\mathcal{O}\left(\frac{\ell(\tau(t))}{(\tau(t))^{\mu}}\right)\right) \quad \text { as } t \rightarrow \infty,
$$

where function $\mathcal{B}(s)$ is defined in (11).

Remark 3. Further reasoning imply that functions $\mathcal{L}(x)$ and $\ell(x)$ can be omitted in estimations of error terms of asymptotic relations in given above Theorems. Taking into account assertions $\left[C_{\mathcal{L}}\right]$ and $\left[C_{\ell}\right]$, these functions are asymptotically constant.

\section{Auxiliaries}

In this section, some auxiliary assertions are provided. They are essential for the proof of theorems.

First, the asymptotic representation of GF of Markov branching processes $Z(t)$ without immigration is considered. Let $F(t ; s)=\mathbb{E}\left[s^{Z(t)} \mid Z(0)=1\right]$ be GF of the process initiated by single individual. Let $R(t ; s):=1-F(t ; s)$. The following result called the Basic lemma of the theory of critical Markov branching processes [4]. It is presented in slightly different form below.

Lemma 1. If condition $\left[f_{\nu}\right]$ holds then

$$
\frac{1}{R(t ; s)}=\frac{(\nu t)^{1 / \nu}}{\mathcal{N}(t)} \cdot\left[1+\frac{\mathcal{M}(s)}{t}\right]^{1 / \nu}
$$


for all $s \in[0,1)$, where $\mathcal{N}(x)$ is $S V_{\infty}$ such that

$$
\mathcal{N}^{\nu}(t) \cdot \mathcal{L}\left(\frac{(\nu t)^{1 / \nu}}{\mathcal{N}(t)}\right) \longrightarrow 1 \text { as } t \rightarrow \infty
$$

and $\mathcal{M}(s)$ is $G F$ of invariant measures of $M B P$ that has the form

$$
\mathcal{M}(s)=\int_{1}^{1 /(1-s)} \frac{d x}{x^{1-\nu} \mathcal{L}(x)} .
$$

Let us introduce the following function

$$
\Lambda(y):=y^{\nu} \mathcal{L}\left(\frac{1}{y}\right)=\frac{f(1-y)}{y}
$$

for $y \in(0,1]$. Let us note that function $y \Lambda(y)$ is positive, tends to zero and it has the monotone derivative so that $y \Lambda^{\prime}(y) / \Lambda(y) \rightarrow \nu$ as $y \downarrow 0$ (see [2, p. 401]). Then it is natural to write

$$
\frac{y \Lambda^{\prime}(y)}{\Lambda(y)}=\nu+\delta(y)
$$

where $\delta(y)$ is continuous and $\delta(y) \rightarrow 0$ as $y \downarrow 0$. Since $\Lambda(1)=\mathcal{L}(1)=a_{0}$ it follows from (14) that

$$
\Lambda(y)=a_{0} y^{\nu} \exp \int_{1}^{y} \frac{\delta(u)}{u} d u .
$$

Therefore

$$
\mathcal{L}\left(\frac{1}{y}\right)=a_{0} \exp \int_{1}^{y} \frac{\delta(u)}{u} d u
$$

Substituting $u=1 / t$ in last integrand, one can obtain

$$
\mathcal{L}(x)=a_{0} \exp \int_{1}^{x} \frac{\varepsilon(t)}{t} d t,
$$

$* * * * * * * * * * * * * * * * * * * * * * * * * * * * * * * * * * * * * * * * * * * * * * * * * * * * * * * * * * * * * * * * * * * * * *$ where $\varepsilon(t)=$ $-\delta(1 / t)$ and $\varepsilon(t) \rightarrow 0$ as $t \rightarrow \infty$. Considering the last equation together with $\left[\mathcal{L}_{\nu}\right]$, one can obtain

$$
\int_{x}^{\lambda x} \frac{\varepsilon(t)}{t} d t=\ln [1+\mathcal{O}(\alpha(x))]=\mathcal{O}(\alpha(x)) \quad \text { as } x \rightarrow \infty
$$

for each $\lambda>0$. Applying the mean value theorem to the left-hand side of the last equality, we have that $\varepsilon(x)=\mathcal{O}(\alpha(x))$. Then condition $\left[\mathcal{L}_{\nu}\right]$ gives

$$
\delta(y)=\mathcal{O}\left(\alpha\left(\frac{1}{y}\right)\right) \quad \text { as } y \downarrow 0 .
$$

The following result is a modification of Lemma 1 and it is required in the subsequent discussions.

Lemma 2. Let assumptions $\left[f_{\nu}\right]$ and $\left[\mathcal{L}_{\nu}\right]$ hold. Then

$$
\frac{1}{\Lambda(R(t ; s))}-\frac{1}{\Lambda(1-s)}=\nu t+\mathcal{O}(\ln \nu(t ; s)) \quad \text { as } t \rightarrow \infty,
$$

where $\nu(t ; s)=\Lambda(1-s) \nu t+1$. 
Proof. One can write from (14) that

$$
\frac{R \Lambda^{\prime}(R)}{\Lambda(R)}=\nu+\delta(R)
$$

since $R:=R(t ; s) \rightarrow 0$ as $t \rightarrow \infty$. Using the backward Kolmogorov equation $\partial F / \partial t=f(F)$ and considering representation $\left[f_{\nu}\right]$, relation (17) becomes

$$
\frac{d \Lambda(R)}{d t}=-\frac{\Lambda(R)}{R} f(1-R)(\nu+\delta(R))=-\Lambda^{2}(R)(\nu+\delta(R)) .
$$

Therefore

$$
d\left[\frac{1}{\Lambda(R)}-\nu t\right]=\delta(R) d t .
$$

Integrating (18) over $[0, t)$, the following equation is obtained

$$
\frac{1}{\Lambda(R(t ; s))}-\frac{1}{\Lambda(1-s)}=\nu t+\int_{0}^{t} \delta(R(u ; s)) d u
$$

where $\delta(y)$ is in (14). Now one should take integral in (19). Considering (15),one can write

$$
\int_{0}^{t} \delta(R(u ; s)) d u=\int_{0}^{t} \mathcal{O}(\Lambda(R(u ; s))) d u .
$$

One should mention that due to (12) $R(t ; s) \rightarrow 0$ as $t \rightarrow \infty$ uniformly in $s \in[0,1)$. Therefore, since $\Lambda(y) \rightarrow 0$ as $y \downarrow 0$, the integral in the right-hand side of $(20)$ is $o(t)$ as $t \rightarrow \infty$. Hence

$$
\Lambda(R(t ; s))=\frac{1}{\lambda(t ; s)}+o\left(\frac{1}{\lambda(t ; s)}\right) \text { as } t \rightarrow \infty,
$$

where $\lambda(t ; s)=\nu t+\Lambda^{-1}(1-s)$. Therefore

$$
\int_{0}^{t} \mathcal{O}(\Lambda(R(u ; s))) d u=\mathcal{O}\left(\int_{0}^{t} \Lambda(R(u ; s)) d u\right)=\mathcal{O}(\ln \nu(t ; s)) \quad \text { as } t \rightarrow \infty .
$$

Together with (19) and (20) this gives relation (16).

Lemma 3. Let $L(t)$ be $S V_{\infty}$ with remainder $\varrho(t)$. Then for $\sigma>0$

$$
\int_{t}^{\infty} y^{-(1+\sigma)} L(y) d y=\frac{1}{\sigma} \frac{1}{t^{\sigma}} L(t)(1+\mathcal{O}(\varrho(t))) \quad \text { as } t \rightarrow \infty .
$$

Proof. Undoubtedly $\int_{1}^{\infty} u^{-(1+\sigma)} d u=1 / \sigma$. Considering this fact and making the substitution $y:=u t$ in the integrand of (21), one can write

$$
\int_{t}^{\infty} y^{-(1+\sigma)} L(y) d y=\frac{1}{\sigma} \frac{L(t)}{t^{\sigma}}\left[1+\sigma \int_{1}^{\infty}\left[\frac{L(u t)}{L(t)}-1\right] u^{-(1+\sigma)} d u\right] .
$$

By definition of $\mathrm{SV}_{\infty}$-function with remainder, the expression in brackets of the integrand on the right-hand side of (22) tends to 0 as $t \rightarrow \infty$ uniformly in $u>1$ (by Uniform Convergence Theorem for $\mathrm{SV}_{\infty}$-functions [2, Theorem 1.5.2]) with the rate $\mathcal{O}(\varrho(t))$. Thus relation (21) is obtained.

The Lemma is proved. 
Lemma 4. Let conditions $\left[\mathcal{L}_{\nu}\right]$ and $\left[\ell_{\delta}\right]$ hold and $\gamma>0$. Then

$$
\int_{x}^{1} \frac{g(u)}{f(u)} d u=\frac{1}{\gamma} \frac{g(x)}{\Lambda(1-x)}(1+\mathcal{O}(\Lambda(1-x))) \quad \text { as } \quad x \uparrow 1 .
$$

Proof. It follows from Basic assumption that

$$
\mathcal{I}(x):=\int_{x}^{1} \frac{g(u)}{f(u)} d u=-\int_{1 /(1-x)}^{\infty} y^{-(1+\gamma)} L(y) d y,
$$

where $L(t)=\ell(t) / \mathcal{L}(t)$ as before. One can easily show that

$$
\frac{L(u t)}{L(t)}-1=\mathcal{O}\left(\frac{\mathcal{L}(t)}{t^{\nu}}\right) \quad \text { as } t \rightarrow \infty
$$

uniformly in $u>0$. Considering the right-hand side of (24), one can directly use (21) with $t=1 /(1-x)$ and $r(t)=\mathcal{O}\left(\mathcal{L}(t) / t^{\nu}\right)$. Then

$$
\mathcal{I}(x)=-\frac{1}{\gamma} \frac{L(t)}{t^{\gamma}}\left[1+\mathcal{O}\left(\frac{\mathcal{L}(t)}{t^{\nu}}\right)\right] \quad \text { as } t \rightarrow \infty .
$$

Now returning to primary designations, relation (23) is obtained.

The Lemma is proved.

\section{Proof of Theorems}

In this final section the Main results are consistently proved.

Proof of Theorem 2.1. Let us rewrite (2) as follows

$$
\mathcal{P}(t ; s)=U(s) \exp \left\{\int_{1}^{F(t ; s)} \frac{g(u)}{f(u)} d u\right\},
$$

where

$$
U(s)=\exp \left\{\int_{s}^{1} \frac{g(u)}{f(u)} d u\right\} .
$$

Considering (3), the integral in (25) converges for $s \in[0,1)$ and becomes 0 as $t \rightarrow \infty$. Therefore $\mathcal{P}(t ; s)$ converges to $U(s)$ as $t \rightarrow \infty$ uniformly over compact subsets. Now, using the functional equation $F(t+\tau ; s)=F(t ; F(\tau ; s))$ (see [9, p. 134]), it follows that

$$
\begin{aligned}
\mathcal{P}(t+\tau ; s) & =\mathcal{P}(\tau ; s) \cdot \exp \left\{\int_{\tau}^{t+\tau} g(F(u ; s)) d u\right\} \\
& =\mathcal{P}(\tau ; s) \cdot \exp \left\{\int_{0}^{t} g(F(u ; F(\tau ; s))) d u\right\}=\mathcal{P}(\tau ; s) \cdot \mathcal{P}(t ; F(\tau ; s)) .
\end{aligned}
$$

Taking limit as $t \rightarrow \infty$, one can obtain the following Schröder type functional equation

$$
U(F(\tau ; s))=\frac{1}{\mathcal{P}(\tau ; s)} U(s) \quad \text { for any } \tau \in \mathcal{T} .
$$


Writing the power series expansion $U(s)=\sum_{j \in \mathcal{S}} u_{j} s^{j}$, equation (27) has an invariant property $u_{j}=\sum_{i \in \mathcal{S}} u_{i} p_{i j}(\tau)$. Obviously $U(1-)=1$ and hence the function in (26) generates an invariant distribution $\left\{u_{j}, j \in \mathcal{S}\right\}$ for MBPI.

Let us prove now of of (4). Considering (25) and using (23), one can obtain

$$
\mathcal{P}(t ; s)=U(s) \exp \{-I(t ; s)\}
$$

where

$$
I(t ; s)=\frac{1}{\gamma} \frac{g(F(t ; s))}{\Lambda(R(t ; s))}(1+\mathcal{O}(\Lambda(R(t ; s)))) \quad \text { as } t \rightarrow \infty .
$$

Next, let us use the asymptotic expansion of $R(t ; s)$. Relation (16) implies

$$
\frac{1}{\Lambda(R(t ; s))}=\lambda(t ; s)\left(1+\mathcal{O}\left(\frac{\ln [\Lambda(1-s) \lambda(t ; s)]}{\lambda(t ; s)}\right)\right) \quad \text { as } t \rightarrow \infty
$$

and therefore

$$
R(t ; s)=\frac{\mathcal{N}(t ; s)}{(\lambda(t ; s))^{1 / \nu}}\left(1+\mathcal{O}\left(\frac{\ln [\Lambda(1-s) \lambda(t ; s)]}{\lambda(t ; s)}\right)\right) \quad \text { as } t \rightarrow \infty,
$$

where $\lambda(t ; s)=\nu t+\Lambda^{-1}(1-s)$ and $\mathcal{N}(t ; s)=\mathcal{L}^{-1 / \nu}(1 / R(t ; s))$. Let us note that $g(s)$ has the form of $\left[g_{\delta}\right]$. Using (30) and (31), one can obtain

$$
\frac{g(F(t ; s))}{\Lambda(R(t ; s))}=-\frac{\mathcal{N}^{\delta}(t ; s)}{(\lambda(t ; s))^{\gamma / \nu}} \ell\left(\frac{1}{R(t ; s)}\right)\left(1+\mathcal{O}\left(\frac{\ln [\Lambda(1-s) \lambda(t ; s)]}{\lambda(t ; s)}\right)\right)
$$

as $t \rightarrow \infty$. It is easy to verify that the function $\mathcal{N}(t ; s)$ is asymptotically equivalent to the $\mathrm{SV}_{\infty}$-function $\mathcal{N}(t)$ defined in Lemma 1.

Asymptotic formula (4) now follows from a combination of (28), (29) and (32). Equation (5) follows from the continuity theorem for power series.

The Theorem is proved.

Proof of Theorem 2.2. Let us write

$$
\begin{aligned}
e^{T(t)} \mathcal{P}(t ; s) & =\exp \left\{(\tau(t))^{|\gamma|}+\int_{0}^{t} g(F(u ; s)) d u\right\}= \\
& =\exp \left\{\Delta(t ; s)+(\tau(t ; s))^{|\gamma|}+\int_{s}^{F(t ; s)} \frac{g(x)}{f(x)} d x\right\},
\end{aligned}
$$

where $\Delta(t ; s)=(\tau(t))^{|\gamma|}-(\tau(t ; s))^{|\gamma|}$ and $\tau(t ; s)=R^{-1}(t ; s)$. Standard integration yields

$$
(\tau(t ; s))^{|\gamma|}=\frac{1}{(1-s)^{|\gamma|}}+\int_{s}^{F(t ; s)} \frac{|\gamma|}{(1-u)^{1+|\gamma|}} d u .
$$

Therefore, relation (33) can be written as follows

$$
e^{T(t)} \mathcal{P}(t ; s)=\pi(s) \cdot \exp \left\{\Delta(t ; s)-\int_{F(t ; s)}^{1}\left[\frac{g(u)}{f(u)}+\frac{|\gamma|}{(1-u)^{1+|\gamma|}}\right] d u\right\}
$$


where $\pi(s)$ has the form of (7). An exponential factor in (34) defines the convergence rate $\rho(t ; s)$ in (8). Let us first evaluate $\Delta(t ; s)$ as $t \rightarrow \infty$. According to Lemma $1 \mathcal{M}(0)=0$ and,therefore, $\tau(t)=\tau(t ; 0)$. Hence, asymptotic representation (12) gives

$$
\begin{aligned}
\Delta(t ; s) & =(\tau(t))^{|\gamma|}\left[1-\left(1+\frac{\mathcal{M}(s)}{t}\right)^{|\gamma| / \nu}\right] \\
& \sim-|\gamma|(\tau(t))^{|\gamma|} \frac{\mathcal{M}(s)}{\nu t}=-|\gamma| \frac{\mathcal{M}(s)}{(\nu t)^{\delta / \nu} \mathcal{N}|\gamma|(t)} \quad \text { as } t \rightarrow \infty .
\end{aligned}
$$

On the other hand $\mathcal{M}(s)$ is bounded for $s \in[0, r], r<1$. Thus

$$
\Delta(t ; s)=\mathcal{O}\left(\frac{\mathcal{L}_{\gamma}(t)}{t^{\delta / \nu}}\right) \quad \text { as } t \rightarrow \infty,
$$

uniformly in $s \in[0, r], r<1$, where $\mathcal{L}_{\gamma}(t)=\mathcal{N}^{-|\gamma|}(t)$.

Let us observe the integral in (34). Taking into account relations (3) and (6), the integrand in brackets becomes $\mathcal{O}\left((1-u)^{\mu-1} \ell(1 /(1-u))\right)$ in the neighbourhood of the point $u=1$. Let us examine the integral $\int_{F(t ; s)}^{1}\left[(1-u)^{\mu-1} \ell(1 /(1-u))\right] d u$ as $t \rightarrow \infty$. Substitution $y=(1-u)^{-1}$ gives the alternative form

$$
\int_{1 / R(t ; s)}^{\infty} y^{-(1+\mu)} \ell(y) d y .
$$

The direct application of Lemma 3 transforms the last integral to the form

$$
\int_{1 / R(t ; s)}^{\infty} y^{-(1+\mu)} \ell(y) d y=\frac{1}{\mu} R^{\mu}(t ; s) \ell\left(\frac{1}{R(t ; s)}\right)(1+o(1)) \quad \text { as } t \rightarrow \infty .
$$

But $R(t ; s)=\tau^{-1}(t ; s)$ and $\tau(t ; s) \tau^{-1}(t) \rightarrow 1$ as $t \rightarrow \infty$ uniformly in $s \in[0,1)$. Thus

$$
\int_{F(t ; s)}^{1}\left[\frac{g(u)}{f(u)}+\frac{|\gamma|}{(1-u)^{1+|\gamma|}}\right] d u=\mathcal{O}\left(\frac{\ell(\tau(t))}{(\tau(t))^{\mu}}\right) \quad \text { as } t \rightarrow \infty .
$$

Taking into account that $\mu<\delta$ and comparing relations (35) and (36), one can obtain that $\Delta(t ; s)$ decreases to zero faster than last integral, i.e., $\Delta(t ; s)=o\left(\ell(\tau(t)) /(\tau(t))^{\mu}\right)$ as $t \rightarrow \infty$. So, asymptotic relation (8) with the error part $\rho(t ; s)$ in form (9) is found from (34)-(36). Equation (10) follows from the continuity theorem for power series.

Finally, one can verify that function $\pi(s)$ satisfies equation (14). Therefore, denoting its power series representation by $\pi(s)=\sum_{j \in \mathcal{S}} \pi_{j} s^{j}$, an invariant property $\pi_{j}=\sum_{i \in \mathcal{S}} \pi_{i} p_{i j}(\tau)$ is obtained for any $\tau>0$. Thus $\left\{\pi_{j}, j \in \mathcal{S}\right\}$ is an invariant measure for MBPI $X(t)$.

The Theorem is proved.

Proof of Corollary 1. The statement follows immediately from (8) by setting $x=0$.

The author is deeply grateful to the anonymous referee for his careful reading of the manuscript and for his kindly comments which contributed to improving the paper. 


\title{
References
}

[1] K.B.Athreya, P.E.Ney, Branching processes, Springer, New York, 1972.

[2] N.H.Bingham, C.M.Goldie, J.L.Teugels, Regular Variation, Cambridge University Press, 1987.

[3] A.A.Imomov, A.Kh.Meyliev, On asymptotic structure of continuous-time MarkovBranching Processes allowing Immigration and withouthigh-order moments, 2020, ArXiv.org/abs/2006.09857v1.

[4] A.A.Imomov, On Conditioned Limit Structure of the Markov Branching Process without Finite Second Moment, Malaysian Journal of Mathematical Sciences, 11(2017), no. 3, 393-422.

[5] A.A.Imomov, On long-term behavior of continuous-time Markov branching processes allowing immigration, Journal of Siberian Federal University. Mathematics and Physics, $\mathbf{7}(2014)$, no. $4,443-454$.

[6] A.A.Imomov, On Markov analogue of Q-processes with continuous time, Theory of Probability and Mathematical Statistics, 84(2012), 57-64. DOI: 10.1090/S0094-9000-2012-00853-3

[7] J.Li, A.Chen, A.G.Pakes, Asymptotic properties of the Markov Branching Process with Immigration, Journal of Theoretical Probability, 25(2012), 122-143.

[8] A.G.Pakes, Revisiting conditional limit theorems for the mortal simple branching process, Bernoulli, 5(1999), no. 6, 969-998.

[9] A.G.Pakes, On Markov branching processes with immigration, Sankhyā: The Indian Journal of Statistics, A37(1975), 129-138.

[10] E.Seneta, Regularly Varying Functions, Springer, Berlin, 1976.

[11] B.A.Sevastyanov, Branching processes, Nauka, Moscow, 1971 (in Russian).

\section{Об оценке скорости сходимости к инвариантным мерам в марковских ветвящихся процессах с возможной бесконечной дисперсией и иммиграцией}

\author{
Азам А. Имомов \\ Каршинский государственный университет \\ Карши, Узбекистан
}

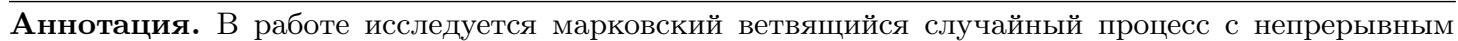
временем и с иммиграцией. Мы рассматриваем критический случай, в котором второй момент закона размножения частиц и первый момент закона иммиграции бесконечны. Предполагая, что нелинейные части соответствующих производящих функций правильно меняются в смысле Карамата, мы доказываем теоремы о сходимости переходных вероятностей процесса к инвариантным мерам. Мы определим скорости этой сходимости при условии, что медленно меняющиеся части являются функциями с остатком.
\end{abstract}

Ключевые слова: марковский ветвящийся процесс, производящие функции, иммиграция, переходные вероятности, медленно меняющаяся функция, инвариантные меры, скорость сходимости. 